Review Article

\title{
Exosome-Derived Noncoding RNAs as a Promising Treatment of Bone Regeneration
}

\author{
Bei Yin (D), Qingge Ma, ${ }^{1,2}$ Chenghao Song, ${ }^{1,2}$ Lingyi Zhao,, ${ }^{1,2}$ Fanyuan Yu,,2 \\ Chenglin Wang, ${ }^{1,2}$ Yu Shi, ${ }^{1}$ and Ling Ye $\mathbb{i}^{1,2}$ \\ ${ }^{1}$ State Key Laboratory of Oral Diseases \& National Clinical Research Center for Oral Diseases, West China Hospital of Stomatology, \\ Sichuan University, Chengdu, China \\ ${ }^{2}$ Department of Endodontics, West China Hospital of Stomatology, Sichuan University, Chengdu, China
}

Correspondence should be addressed to Ling Ye; ling-ye@hotmail.com

Received 15 October 2020; Revised 18 December 2020; Accepted 7 January 2021; Published 21 January 2021

Academic Editor: Stefan Arnhold

Copyright ( $) 2021$ Bei Yin et al. This is an open access article distributed under the Creative Commons Attribution License, which permits unrestricted use, distribution, and reproduction in any medium, provided the original work is properly cited.

\begin{abstract}
The reconstruction of large bone defects remains a crucial challenge in orthopedic surgery. The current treatments including autologous and allogenic bone grafting and bioactive materials have their respective drawbacks. While mesenchymal stem cell (MSC) therapy may address these limitations, growing researches have demonstrated that the effectiveness of MSC therapy depends on paracrine factors, particularly exosomes. This aroused great focus on the exosome-based cell-free therapy in the treatment of bone defects. Exosomes can transfer various cargoes, and noncoding RNAs are the most widely studied cargo through which exosomes exert their ability of osteoinduction. Here, we review the research status of the exosome-derived noncoding RNAs in bone regeneration, the potential application of exosomes, and the existing challenges.
\end{abstract}

\section{Current Status of Bone Regeneration}

The reconstruction of large bone defects is a key challenge in reconstructive surgery. Currently, the treatment strategies include autologous and allogenic bone grafting, bioactive materials. However, they all have their limitations in various aspects. Autologous bone grafts have the disadvantages of limited sources of bone and extra surgical injuries. Allogenic bone grafts may cause immunological rejection and disease transmission. As for the bioactive materials, the issues of biocompatibility, structural stability, mechanical strength, and degradability remain to be solved.

Stem cell-based engineered bone may address the limitations of the abovementioned methods. Nevertheless, the satisfactory results are hindered by MSCs' limited ability to form enough new bone. Therefore, approaches of enhancing MSC's osteogenic differentiation have been investigated such as gene editing $[1,2]$, the use of growth factors [3], and the addition of cell-derived conditioned medium $(\mathrm{CM})[4,5]$. However, several considerations need to be clarified in terms of cell transplantation. For example, genetic modification gives rise to safety issues; the concentration of biotherapeutics in CM is low, and CM may contain medium contaminants. Moreover, stem cell therapy is further hindered by insufficient cell number, complex and costly expansion procedures, immunological rejection, the accumulation of genomic alterations [6], the risk of tumor [7], the formation of emboli [8], and so forth.

\section{Exosomes}

In recent years, accumulating researches demonstrated that the paracrine role may account for the efficacy of MSC therapy given that limited cells have engrafted into the sites of injury $[9,10]$ and that bone regeneration can be regulated by paracrine factors $[11,12]$. In this scenario, exosome transplantation is considered as a novel cell-free therapy for bone regeneration. Exosomes are $40-100 \mathrm{~nm}$ extracellular vesicles (EVs). They are released by multivesicular bodies (MVBs) after fusion with cytomembranes. MVBs are late endosomes with many intraluminal vesicles inside formed by inward budding of endosomal membranes [13]. Exosomes can be 
transported to distant sites via body fluids, or they can be invaginated by residing cells. They exist in a variate of body fluids such as breast milk, saliva, lymph, and bile.

Unlike stem cell therapy, the application of exosomes involves fewer safety considerations. In fact, several clinical studies have proved the safety of exosomes in the treatment of cancers [14]. Several other registered NIH clinical trials for the treatment of ulcers, diabetes, and oral mucositis are undergoing. To date, MSCs are the most prolific producer of exosomes. Immortalization of MSCs has no effects on the yield or the properties of exosomes, while it compromises the differentiation potential of MSCs [15]. Moreover, exosomes can be engineered to act as the carriers of RNArelated products such as siRNA and shRNA, with enhanced efficacy compared to nanoparticles and liposomes [16, 17]. Because exosomes contain several transmembrane proteins which can promote endocytosis while prevent phagocytosis by monocytes [18]. Furthermore, exosomes have a high degree of stability, in that their potency can be maintained at $-20^{\circ} \mathrm{C}$ for 6 months [19].

\section{Exosomes Regulate Osteogenic Ability of MSCs}

Exosomes can promote the osteogenic ability of MSCs, and the effects of exosomes often increase with increasing concentrations. Indeed, some studies reported that exosomes even outperformed the currently used osteoinductive cocktail, the conditioned medium, and the extracellular matrix (ECM) [20]. In vivo experiments also revealed that exosomes dramatically stimulated osteogenesis in calvarial defects [21], bone fracture [22], and radiation-induced bone loss [23]. Exosomes affect the osteogenic differentiation of recipient cells by regulating various signaling pathways including TGF- $\beta 1$ pathway, Wnt/ $\beta$-catenin pathway [24], and MAPK pathway [25] and by upregulating mRNA and protein expression of osteogenesis-related genes such as Runtrelated transcription factor 2 (RUNX2), Osteocalcin (OC), and Osterix (OSX). While exosomes derived from osteogenic conditions performed better in terms of bone regeneration [26], it is worth noting that exosomes from pathological MSCs such as MSCs from type 1 diabetes and those from the aged even inhibit the osteogenesis [27, 28].

Exosomes exert their influence either through interacting with the extracellular matrix or through internalization into cells. Once released, exosomes can anchor to the ECM and act as the initial sites for mineralized nodule along with matrix vesicles [29]. Recently, increasing researches have focused on the role of exosomes in delivering cargoes by internalization into cells. The various cargoes include proteins, nucleic acid, and lipid. Among them, noncoding RNAs (ncRNAs) are the most widely studied cargoes through which exosomes exert their ability of osteoinduction (Table 1).

\section{Noncoding RNAs}

High-throughput technologies have discovered about $90 \%$ of the genome is actively transcribed [30], but the majority
(98\%) of transcripts exist as ncRNAs. NcRNAs were initially regarded as transcriptional noise, but recent studies found they could exert regulative effects on various biological processes [31]. NcRNAs are divided into two classes based on the size, long ncRNAs (>200 nucleotides, long intergenic ncRNAs, antisense RNAs, etc.) and small ncRNAs $(<200$ nucleotides, including small interfering RNAs (siRNA), microRNAs (miRNAs), etc.).

4.1. MicroRNAs. miRNAs affect the expression of mRNAs by two modes including translational repression and mRNA decay, both of which were realized by the RNA-induced silencing complex (RISC) formed by miRNAs and Argonaute protein (Ago) [32]. When the miRNAs complement perfectly with the $3^{\prime}$ (or $5^{\prime}$ in some cases [33]) - untranslated region ( 3 'UTR) of the mRNAs, mRNA decay occurs through endonucleolytic cleavage by RISC. In the cases of partial complementation, RISC can recruit cofactor proteins to induce mRNA decay or translational repression in a manner independent of endonucleolytic cleavage $[34,35]$. The MSC exosomal miRNAs are enriched in various KEGG pathways: Wnt, MAPK, and PI3K-Akt may be the signaling pathways through which exosomal miRNAs exert their effects [36]. Pathways including endocytosis and actin cytoskeleton are possibly related to the internalization of exosomes. Other pathways such as spliceosome, mRNA surveillance, and RNA transport are possible mechanisms of how miRNAs regulate the target cells [37].

The osteogenic induction of MSCs alters the expression of exosomal miRNAs. Several well-known suppressors of osteogenesis, such as miR-144, miR-31, and miR-221, were downregulated in exosomes from osteogenic differentiated MSCs, while positive regulators of osteogenesis like miR-21 were upregulated [38]. miR-31 and miR-221 can suppress osteogenic differentiation through targeting the 3 ' untranslated regions of Runx 2 and inhibiting Runx 2 gene expression $[39,40]$. miR-144-3p can target DNA demethylase teneleven translocation-2 (TET2), leading to the increase of 5hydroxymethyl-cytosine $(5 \mathrm{hmC})$ levels and decrease of osteogenic genes expression [41]. Connexin-43 and Smad4 can also be targeted by miR-144-3p [42, 43]. miR-21 enhances osteogenic differentiation by downregulating Sox 2 and Smad7 [44, 45].

Exosomal miRNA played an indispensable role in the cross-talk between bone and muscle. Myoblast-derived exosomes could deliver miR-27a-3p to the preosteoblasts and decrease the expression of adenomatous polyposis coli (APC), a negative regulator of $\beta$-catenin, thus, activating the $\beta$-catenin pathway. The effects of exosomes largely relied on miR-27a-3p, given that myoblast exosomes whose miR27a-3p was inactivated lost their osteogenic-inductive capacity [46]. miR-27a-3p may also regulate osteogenic differentiation through targeting activating transcription factor 3 (ATF3). ATF3 can bind to the promoter of ALP and negatively regulate ALP expression [47].

Preosteoblast-derived exosomes contain miRNAs that can regulate the osteogenic differentiation. The preosteoblast exosomes contain abundant let-7 miRNA [48], which is a 


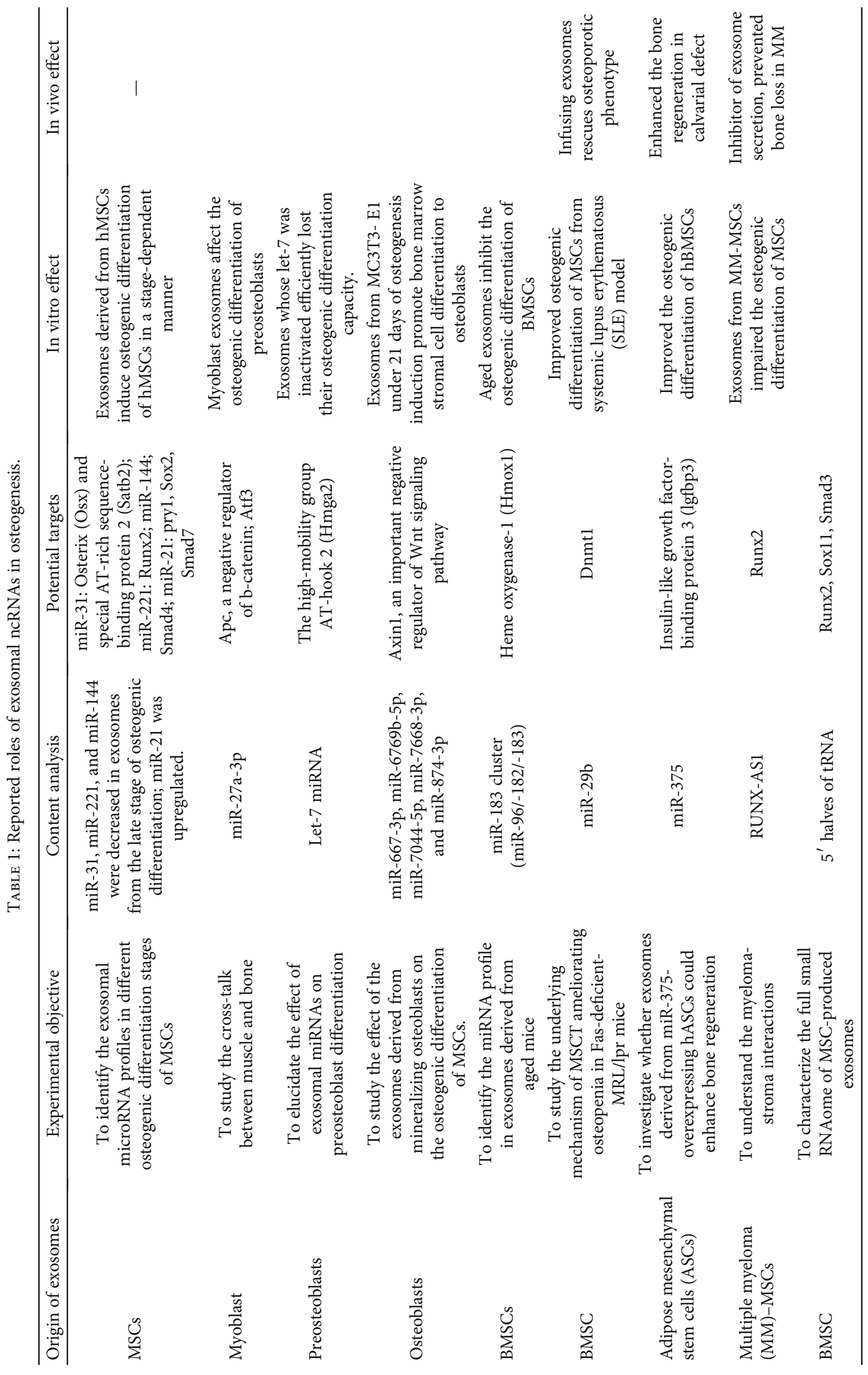


pivotal regulator of osteogenesis by targeting the highmobility group AT-hook 2 (HMGA2) [49]. Exosomes from MC3T3-E1 cells facilitated the osteogenesis of bone marrow stromal ST2 cells. Through a study of the miRNA profile in ST2 cells and that in MC3T3-E1 exosomes, they identified several miRNAs (miR-7668-3p, miR-667-3p, miR-7044-5p, and $\mathrm{miR}-874-3 \mathrm{p}$ ) that were transferred from preosteoblast exosomes to bone marrow stromal cells [50]. All the above miRNAs target Axin1, a suppressor of the Wnt signaling pathway, to exert their osteoinductive effects [51, 52].

In aging mice, the miRNA profile of exosomes was quite different from those in young mice. Specifically, the miR-183 cluster is enriched in aged exosomes [27]. miR-183-5p could increase the expression of the senescence marker $\beta$-galactosidase and suppress the osteogenic differentiation of BMSCs. Heme oxygenase-1 (Hmox1) is also a target of miR-183-5p, which has been shown previously to stimulate BMSCs osteogenic differentiation [53].

Apart from transporting microRNAs directly, exosomes can also transport protein to affect miRNA expression indirectly in recipient cells. In systemic lupus erythematosus (SLE) model Fas-deficient-MRL/lpr mice, exosome transplantation rescued the osteoporotic phenotype by the Fas/miR-29b/Dnmt1/Notch cascade. Exosome infusion provided donor-derived Fas to recipient cells, which facilitated the release of miR-29b into the extracellular environment and the decrease of intracellular miR-29b [54]. miR-29b decrease led to the upregulation of its direct target, DNA methyltransferase 1 (Dnmt1). Then, Dnmt1 controlled the hypermethylation and thus inactivation of Notch1, which is a negative regulator of osteogenesis [55].

4.2. Antisense lncRNAs. Antisense (AS) lncRNAs have sequences complementary to their sense counterparts. Antisense lncRNAs mainly function through regulating the expression of their sense transcripts. In some cases, they can form an RNA-RNA duplex with their sense counterparts, stabilizing their sense transcripts and thus increasing the gene expression [56, 57]. In other cases, AS lncRNAs mediate transcriptional repression of their sense proteincoding genes [58].

Exosome-derived antisense lncRNAs play a role in affecting the osteogenic ability of MSCs. Multiple myeloma (MM) is a plasma cell cancer characterized by multiple osteolytic damage. In vitro experiments demonstrated myelomaderived exosomes could decrease MSCs' osteogenic differentiation ability. In vivo treatment of GW4869, an inhibitor of exosome secretion, attenuated bone loss in multiple myeloma models, with the expression of bone resorption marker betaisomerized C-telopeptide $(\beta$-CTX) decreased and that of osteogenesis-related gene procollagen type I N-terminal propeptide (P1NP) increased. Using a lncRNA sequencing, they identified that antisense lncRNA RUNX-AS1 was enriched in MM-MSCs and MM-MSC-derived exosomes. Mechanismly, antisense lncRNA RUNX-AS1 and RUNX2 formed a RNA duplex at overlapping regions through base pairing, interfered with RUNX2 pre-mRNA splicing, and suppressed RUNX2 mRNA expression. Thereby, the MM-MSC-derived exosomes may transmit the antisense lncRNA RUNX-AS1 to MSCs, contributing to the impaired osteogenic differentiation ability in MM-MSCs [59].

4.3. Transfer RNA (tRNA) Halves. Mature cytoplasmic tRNAs can be cleavaged into small RNA fragments: the $5^{\prime}$ and $3^{\prime}$ tRNA halves (30-40 nt in size). The $5^{\prime}$ tRNA halves can silence target mRNAs by complementary base pairing to the $3^{\prime}$ UTRs of protein-coding genes in a manner like miRNA/siRNA [60,61]. While previous studies found the $5^{\prime}$ tRNA halves were induced under stress to suppress translation and preserve energy, recent studies found that tRNA halves existed in certain types of cells persistently. Under physiological conditions, the bone marrow is the specific tissue expressing significant quantities of $5^{\prime}$ tRNA halves [62], while their level is quite low in several other tissues. MSC exosomes are abundant with $5^{\prime}$ tRNA halves with the targets of osteogenesis-related genes, such as RUNX2 and SMAD3 [63].

\section{Strategies for Clinical Application of Exosomes}

When it comes to clinical application, the pharmacokinetics of exosomes should be noted. One study showed that the exosomes predominately existed in the bone and lung 24 hours after injection [64]. However, other studies showed that the majority of exosomes were distributed in organs of rich vascular such as kidney, spleen, and lung $[65,66]$. Contrary to systemic administration, local administration can maintain high concentrations of exosomes at target sites. Additionally, exosomes can be anchored to biomaterials/scaffolds, such as fibronectin, type I collagen, hydrogel, tricalcium phosphate, poly-lactic-glycolic acid (PLGA), and hydrogel glue, to support their delivery and to facilitate a controlled release of exosomes while enhancing the osteogenic ability of the biomaterials $[67,68]$. It is worth noting that various aspects of the scaffolds could make influences on the behavior of the combined cells and the exosomes. For example, surface roughness plays a role in regulating both the mechanical strength of the material [69] and cell behavior. Whether the surface of the scaffolds affects the function of exosomes needs further study. To avoid the possible effects made by the heterogeneous morphology of the scaffolds when evaluating biomaterials carried with exosomes, the computer-aided design (CAD) technology may help implement a standardization of the shape and the surface of the scaffolds [70].

The osteogenic capacities of exosomes can be improved by modifying either the parent cells or the exosomes. The modifications include biochemical factors and mechanical factors. In fact, mechanical stimuli such as low-intensity pulsed ultrasound (LIPUS) could be used to enhance the osteoinductive capability of MSCs [71]. Whether the exosomes from the MSCs under such mechanical stimuli played a better role in bone regeneration needs further study. As for the biochemical factors, one study modified parent cells by miR-375-overexpressing, which resulted in a significant increase of miR-375 in MSC exosomes. These exosomes had enhanced abilities of bone regeneration in calvarial 
defects [72]. The exosomes from the TNF- $\alpha$-primed cells had an elevated level of Wnt-3a compared with unprimed cells, contributing to the enhanced osteoinductive effects of exosomes [73]. HIF- $1 \alpha$ can stimulate BMSC osteogenic differentiation and enhance angiogenic cell functions. Li constructed HIF- $1 \alpha$ mutant BMSCs in which HIF- $1 \alpha$ expresses continuously even under normoxic conditions. They found that exosomes from HIF- $1 \alpha$ mutant BMSCs (BMSC-Exos$\mathrm{MoU}$ ) had stronger osteoinductive capacity than those from the wild-type group [74]. Another study reported the exosomes from BMP2-stimulated macrophages integrated to the titanium implants improved the biofunction of the plants by increasing the expression of ALP, BMP2, growth/differentiation factor (GDF)-15, etc. [75]. The therapeutic ability of exosomes can also be improved via loading exosomes with content such as peptides and siRNA by electroporation, which has been studied in disease models of Parkinson's and Alzheimer's [76].

\section{Challenges for Clinical Application of Exosomes}

Despite their great potential in bone regeneration, several challenges existed in the application of MSC-derived exosomes. Among them, the low yield remains to be a major challenge. Several strategies have been explored to maximize yield, including serum starvation and modulating calcium concentration. Nevertheless, those operations could potentially alter the contents and function of exosomes. Another strategy is to increase the supply of MSCs by immortalization. Researchers found that transfection of the c-myc gene provided infinite cell sources for the production of exosomes [77]. However, MYC transformation may give rise to the risks of tumorigenesis, considering that immortalized cells may produce EVs with an altered content or even worse with prooncogenic factors $[78,79]$. Therefore, it is important to find a cell source that is efficient in exosomes production. MSCs are the important source of large-scale production of exosomes. Apart from exosomes derived from BMSCs, dental-derived mesenchymal stem cells (D-dMSCs) exosomes may potentially be an excellent or even superior alternative in terms of bone regeneration, particularly the craniomaxillofacial bone. D-dMSCs are abundant, including dental pulp stem cells (DPSCs), gingival mesenchymal stem cells (GMSCs), periodontal ligament stem cells (PDLSCs), dental follicle progenitors (DFPCs), and periapical cystmesenchymal stem cells (PCy-MSCs). The procedure of harvesting D-dMSCs is noninvasive. Biological "waste" such as orthodontic teeth, the deciduous teeth, and even the periapical inflammatory cystitis can be the smart source of DdMSCs. Among the various kinds of D-dMSCs, PCy-MSCs presented a better capability towards osteogenic commitment $[80,81]$. Whether the exosomes derived from PCyMSCs play a better role in bone regeneration remained unexplored. Future studies are needed to clarify in depth the secretomes of the abovementioned MSCs, aiming to figure out the most effective cell source. Besides, when isolating MSCs such as gingival MSCs, new technologies like bioimpedance assay may potentially help identify the healthy and the early potential lesion [82] to ensure harvesting exosomes from healthy cells.

The target specificity of exosomes needs to be further studied and utilized. The internalization of exosomes is realized through cell-exosome interactions involving transmembrane proteins and ECM proteins $[83,84]$, of which the underlying mechanisms remain unclear. Ligandreceptor recognition may serve a major role in the binding of exosomes to recipient cells. To optimize targeting specificity, antigen or ligands should be developed to attach to the membranes of exosomes. Exosomes loaded with MAGE (melanoma-associated antigen) were used to target the lung cancer cells in a clinical trial (clinicaltrials.gov/NCT01159288). One study used this kind of engineered exosomes to deliver siRNA to the brain. They pretransfected dendritic cells with a plasmid with the neuron-specific RVG peptide clone into the exosomal membrane protein Lamp2b. These exosomes delivered siRNAs specifically to the brain without non-specific delivery and achieved strong silencing of BACE1, a therapeutic target of Alzheimer's disease [85].

The procedures of isolation and administration should be taken into consideration when making conclusions. Important differences occur in terms of the quality and the RNA profiling when using different isolation protocols, such as centrifugation, chromatography, filtration, and polymerbased precipitation [86]. Up to now, there is no consensus on isolation protocols. More standardized methods of preparation should be carried out to get comparable and reliable results.

\section{Concluding Remarks}

The role of exosomes in bone regeneration has been well recognized, and the noncoding RNAs play an important role in exosomes-regulated osteogenic differentiation. If the abovementioned challenges are met, the MSC-derived exosomes for cell-free therapy may offer an elegant alternative for the treatment of bone defects.

\section{Conflicts of Interest}

The authors deny any conflicts of interest.

\section{Acknowledgments}

This study is supported by grants from the National Natural Science Foundation of China (81825005, 82001019, and 81873708).

\section{References}

[1] Y. Kim, B.-J. Kang, W. Kim, H.-s. Yun, and O.-k. Kweon, "Evaluation of mesenchymal stem cell sheets overexpressing BMP-7 in canine critical-sized bone defects," International Journal of Molecular Sciences, vol. 19, no. 7, p. 2073, 2018.

[2] J.-C. Liao, "Cell Therapy Using Bone Marrow-Derived Stem Cell Overexpressing BMP-7 for Degenerative Discs in a Rat Tail Disc Model," International Journal of Molecular Sciences, vol. 17, no. 2, p. 147, 2016. 
[3] W. He, L. Chen, Y. Huang et al., "Synergistic effects of recombinant Lentiviral-mediated BMP2 and TGF-beta 3 on the osteogenic differentiation of rat bone marrow mesenchymal stem cells_in vitro_," Cytokine, vol. 120, pp. 1-8, 2019.

[4] S. Zhong, X. He, Y. Li, and X. Lou, "Conditioned Medium Enhances Osteogenic Differentiation of Induced Pluripotent Stem Cell-Derived Mesenchymal Stem Cells," Tissue Engineering and Regenerative Medicine, vol. 16, no. 2, pp. 141-150, 2019.

[5] W. Chang, R. Kim, S. In Park et al., "Enhanced healing of rat calvarial bone defects with hypoxic conditioned medium from mesenchymal stem cells through increased endogenous stem cell migration via regulation of ICAM-1 targeted-microRNA221," Molecules and Cells, vol. 38, no. 7, pp. 643-650, 2015.

[6] B. G. Stultz, K. McGinnis, E. E. Thompson, J. L. Lo Surdo, S. R. Bauer, and D. A. Hursh, "Chromosomal stability of mesenchymal stromal cells during _in vitro_ culture," Cytotherapy, vol. 18, no. 3, pp. 336-343, 2016.

[7] P. W. Marks, C. M. Witten, and R. M. Califf, "Clarifying stemcell therapy's benefits and risks," New England Journal of Medicine, vol. 376, no. 11, pp. 1007-1009, 2017.

[8] J. W. Jung, M. Kwon, J. C. Choi et al., "Familial occurrence of pulmonary embolism after intravenous, adipose tissue-derived stem cell therapy," Yonsei Medical Journal, vol. 54, no. 5, pp. 1293-1296, 2013.

[9] L. von Bahr, I. Batsis, G. Moll et al., "Analysis of Tissues Following Mesenchymal Stromal Cell Therapy in Humans Indicates Limited Long-Term Engraftment and No Ectopic Tissue Formation," STEM CELLS, vol. 30, no. 7, pp. 15751578, 2012.

[10] J. L. Spees, R. H. Lee, and C. A. Gregory, "Mechanisms of mesenchymal stem/stromal cell function," Stem Cell Research \& Therapy, vol. 7, no. 1, p. 125, 2016.

[11] X. Li, Y. Zheng, L. Hou et al., "Exosomes derived from maxillary BMSCs enhanced the osteogenesis in iliac BMSCs," Oral Diseases, vol. 26, no. 1, pp. 131-144, 2020.

[12] R. Sun, S. Xu, and Z. Wang, "Rat sinus mucosa- and periosteum-derived exosomes accelerate osteogenesis," Journal of Cellular Physiology, vol. 234, no. 12, pp. 21947-21961, 2019.

[13] J. R. Edgar, "Q\&A: What are exosomes, exactly?," BMC Biology, vol. 14, no. 1, 2016.

[14] B. Escudier, T. Dorval, N. Chaput et al., "Vaccination of metastatic melanoma patients with autologous dendritic cell (DC) derived-exosomes: results of the first phase I clinical trial," Journal of Translational Medicine, vol. 3, no. 1, p. 10, 2005.

[15] R. W. Y. Yeo, R. C. Lai, B. Zhang et al., "Mesenchymal stem cell: an efficient mass producer of exosomes for drug delivery," Advanced Drug Delivery Reviews, vol. 65, no. 3, pp. 336-341, 2013.

[16] K. B. Johnsen, J. M. Gudbergsson, M. N. Skov, L. Pilgaard, T. Moos, and M. Duroux, "A comprehensive overview of exosomes as drug delivery vehicles - Endogenous nanocarriers for targeted cancer therapy," Biochimica et Biophysica Acta (BBA)-Reviews on Cancer, vol. 1846, no. 1, pp. 75-87, 2014.

[17] P. Vader, E. A. Mol, G. Pasterkamp, and R. M. Schiffelers, "Extracellular vesicles for drug delivery," Advanced Drug Delivery Reviews, vol. 106, pp. 148-156, 2016.

[18] S. Kamerkar, V. S. LeBleu, H. Sugimoto et al., "Exosomes facilitate therapeutic targeting of oncogenic KRAS in pancreatic cancer," Nature, vol. 546, no. 7659, pp. 498-503, 2017.
[19] B. Yu, X. Zhang, and X. Li, "Exosomes derived from mesenchymal stem cells," International Journal of Molecular Sciences, vol. 15, no. 3, pp. 4142-4157, 2014.

[20] K. Narayanan, S. Kumar, P. Padmanabhan, B. Gulyas, A. C. A. Wan, and V. M. Rajendran, "Lineage-specific exosomes could override extracellular matrix mediated human mesenchymal stem cell differentiation," Biomaterials, vol. 182, pp. 312-322, 2018.

[21] X. Qi, J. Zhang, H. Yuan et al., "Exosomes secreted by humaninduced pluripotent stem cell-derived mesenchymal stem cells repair critical-sized bone defects through enhanced angiogenesis and osteogenesis in osteoporotic rats," International Journal of Biological Sciences, vol. 12, no. 7, pp. 836-849, 2016.

[22] J. Zhang, X. Liu, H. Li et al., "Exosomes/tricalcium phosphate combination scaffolds can enhance bone regeneration by activating the PI3K/Akt signaling pathway," Stem Cell Research Therapy, vol. 7, no. 1, p. 136, 2016.

[23] M. Martins, D. Ribeiro, A. Martins, R. L. Reis, and N. M. Neves, "Extracellular Vesicles Derived from Osteogenically Induced Human Bone Marrow Mesenchymal Stem Cells Can Modulate Lineage Commitment," Stem Cell Reports, vol. 6, no. 3, pp. 284-291, 2016.

[24] R. Zuo, M. Liu, Y. Wang et al., "BM-MSC-derived exosomes alleviate radiation-induced bone loss by restoring the function of recipient BM-MSCs and activating Wnt/ $\beta$-catenin signaling," Stem Cell Research \& Therapy, vol. 10, no. 1, p. 30, 2019.

[25] P. Zhao, L. Xiao, J. Peng, Y.-Q. Qian, and C.-C. Huang, "Exosomes derived from bone marrow mesenchymal stem cells improve osteoporosis through promoting osteoblast proliferation via MAPK pathway," European Review for Medical and Pharmacological Sciences, vol. 22, no. 12, pp. 3962-3970, 2018.

[26] C.-C. Huang, R. Narayanan, S. Alapati, and S. Ravindran, "Exosomes as biomimetic tools for stem cell differentiation: applications in dental pulp tissue regeneration," Biomaterials, vol. 111, pp. 103-115, 2016.

[27] C. Davis, A. Dukes, M. Drewry et al., "MicroRNA-183-5p Increases with Age in Bone-Derived Extracellular Vesicles, Suppresses Bone Marrow Stromal (Stem) Cell Proliferation, and Induces Stem Cell Senescence," Tissue Engineering Part A, vol. 23, no. 21-22, pp. 1231-1240, 2017.

[28] Y. Zhu, Y. Jia, Y. Wang, J. Xu, and Y. Chai, "Impaired Bone Regenerative Effect of Exosomes Derived from Bone Marrow Mesenchymal Stem Cells in Type 1 Diabetes," STEM CELLS Translational Medicine, vol. 8, no. 6, pp. 593-605, 2019.

[29] I. M. Shapiro, W. J. Landis, and M. V. Risbud, "Matrix vesicles: are they anchored exosomes?," Bone, vol. 79, pp. 29-36, 2015.

[30] L. D. Stein, "End of the beginning," Nature, vol. 431, no. 7011, pp. 915-916, 2004.

[31] A. Fatica and I. Bozzoni, "Long non-coding RNAs: new players in cell differentiation and development," Nature Reviews Genetics, vol. 15, no. 1, pp. 7-21, 2014.

[32] C.-C. Chang, M. T. Venø, L. Chen et al., "Global MicroRNA Profiling in Human Bone Marrow Skeletal-Stromal or Mesenchymal-Stem Cells Identified Candidates for Bone Regeneration," Molecular Therapy, vol. 26, no. 2, pp. 593605,2018

[33] Z. Bai, Y. Huang, W. Li et al., "Genomewide mapping and screening of Kaposi's sarcoma-associated herpesvirus (KSHV) 3' untranslated regions identify bicistronic and polycistronic viral transcripts as frequent targets of KSHV microRNAs," Journal of Virology, vol. 88, no. 1, pp. 377-392, 2013. 
[34] S. Jonas and E. Izaurralde, "Towards a molecular understanding of microRNA-mediated gene silencing," Nature Reviews Genetics, vol. 16, no. 7, pp. 421-433, 2015.

[35] H. Iwakawa and Y. Tomari, "The functions of microRNAs: mRNA decay and translational repression," Trends in Cell Biology, vol. 25, no. 11, pp. 651-665, 2015.

[36] S. Yang, S. Guo, S. Tong, and X. Sun, "Promoting Osteogenic Differentiation of Human Adipose-Derived Stem Cells by Altering the Expression of Exosomal miRNA," Stem Cells International, vol. 2019, Article ID 1351860, 15 pages, 2019.

[37] J.-F. Xu, G.-h. Yang, X.-H. Pan et al., "Altered microRNA expression profile in exosomes during osteogenic differentiation of human bone marrow-derived mesenchymal stem cells," PLoS ONE, vol. 9, no. 12, 2014.

[38] X. Wang, O. Omar, F. Vazirisani, P. Thomsen, and K. Ekström, "Mesenchymal stem cell-derived exosomes have altered microRNA profiles and induce osteogenic differentiation depending on the stage of differentiation," PLOS ONE, vol. 13, no. 2, p. e0193059, 2018.

[39] L. Yu, Y. Xu, H. Qu et al., "Decrease of MiR-31 induced by TNF- $\alpha$ inhibitor activates SATB2/RUNX2 pathway and promotes osteogenic differentiation in ethanol-induced osteonecrosis," Journal of Cellular Physiology, vol. 234, no. 4, pp. 4314-4326, 2019.

[40] Y. Deng, S. Wu, H. Zhou et al., "Effects of a miR-31, Runx2, and Satb2 Regulatory Loop on the Osteogenic Differentiation of Bone Mesenchymal Stem Cells," Stem Cells and Development, vol. 22, no. 16, pp. 2278-2286, 2013.

[41] N. Li, L. Liu, Y. Liu, S. Luo, Y. Song, and B. Fang, "miR-1443p Suppresses Osteogenic Differentiation of BMSCs from Patients with Aplastic Anemia through Repression of TET2," Molecular Therapy - Nucleic Acids, vol. 19, pp. 619626, 2020.

[42] Z. Sun, F. Wu, Y. Yang et al., "MiR-144-3p Inhibits BMSC Proliferation and Osteogenic DifferentiationViaTargeting FZD4 in Steroid-Associated Osteonecrosis," Current Pharmaceutical Design, vol. 25, no. 45, pp. 4806-4812, 2020.

[43] C. Huang, J. Geng, X. Wei, R. Zhang, and S. Jiang, "MiR-144$3 p$ regulates osteogenic differentiation and proliferation of murine mesenchymal stem cells by specifically targetingsmad4," FEBS Letters, vol. 590, no. 6, pp. 795-807, 2016.

[44] Q. Song, L. Zhong, C. Chen et al., "MiR-21 synergizes with BMP9 in osteogenic differentiation by activating the BMP9/Smad signaling pathway in murine multilineage cells," International Journal of Molecular Medicine, vol. 36, no. 6, pp. 1497-1506, 2015.

[45] O. Trohatou, D. Zagoura, V. Bitsika et al., "Sox2 suppression by miR-21 governs human mesenchymal stem cell properties," Stem Cells Translational Medicine, vol. 3, no. 1, pp. 54-68, 2014.

[46] Q. Xu, Y. Cui, J. Luan, X. Zhou, H. Li, and J. Han, "Exosomes from $\mathrm{C} 2 \mathrm{C} 12$ myoblasts enhance osteogenic differentiation of MC3T3-E1 pre-osteoblasts by delivering miR-27a-3p," Biochemical and Biophysical Research Communications, vol. 498, no. 1, pp. 32-37, 2018.

[47] Y. C. Fu, S. R. Zhao, B. H. Zhu, S. S. Guo, and X. X. Wang, "MiRNA-27a-3p promotes osteogenic differentiation of human mesenchymal stem cells through targeting ATF3," European Review for Medical and Pharmacological Sciences, vol. 23, suppl. 3, pp. 73-80, 2019.

[48] S.-Y. Choi, E.-C. Han, S.-H. Hong, T.-G. Kwon, Y. Lee, and H.J. Lee, "Regulating Osteogenic Differentiation by Suppression of Exosomal MicroRNAs," Tissue Engineering Part A, vol. 25, no. 15-16, pp. 1146-1154, 2019.

[49] J. Wei, H. Li, S. Wang et al., "let-7Enhances osteogenesis and bone formation while repressing adipogenesis of human stromal/mesenchymal stem cells by regulating HMGA2," Stem Cells and Development, vol. 23, no. 13, pp. 14521463, 2014.

[50] Y. Cui, J. Luan, H. Li, X. Zhou, and J. Han, "Exosomes derived from mineralizing osteoblasts promote ST2 cell osteogenic differentiation by alteration of microRNA expression," FEBS Letters, vol. 590, no. 1, pp. 185-192, 2016.

[51] S. Park, M.-S. Lee, J. Gwak et al., "CCAAT/enhancer-binding protein- $\beta$ functions as a negative regulator of $\mathrm{Wnt} / \beta$-catenin signaling through activation of _AXIN1_ gene expression," Cell Death \& Disease, vol. 9, no. 10, p. 1023, 2018.

[52] L. Ji, B. Lu, R. Zamponi et al., "USP7 inhibits Wnt/ $\beta$-catenin signaling through promoting stabilization of Axin," Nature Communications, vol. 10, no. 1, p. 4184, 2019.

[53] K. Ke, O.-J. Sul, M. Rajasekaran, and H.-S. Choi, "MicroRNA183 increases osteoclastogenesis by repressing heme oxygenase-1," Bone, vol. 81, pp. 237-246, 2015.

[54] S. Liu, D. Liu, C. Chen et al., "MSC Transplantation Improves Osteopenia via Epigenetic Regulation of Notch Signaling in Lupus," Cell Metabolism, vol. 22, no. 4, pp. 606618, 2015.

[55] Z. Luo, X. Shang, H. Zhang et al., "Notch signaling in osteogenesis, osteoclastogenesis, and angiogenesis," The American Journal of Pathology, vol. 189, no. 8, pp. 1495-1500, 2019.

[56] I. Amit-Avraham, G. Pozner, S. Eshar et al., "Antisense long noncoding RNAs regulate var gene activation in the malaria parasite Plasmodium falciparum," Proceedings of the National Academy of Sciences, vol. 112, no. 9, pp. e982-e991, 2015.

[57] F. Wang, R. Liang, B. Soibam, J. Yang, and Y. Liu, "Coregulatory long non-coding RNA and protein-coding genes in serum starved cells," Biochimica et Biophysica Acta (BBA)-Gene Regulatory Mechanisms, vol. 1862, no. 1, pp. 84-95, 2019.

[58] J. D. Resnick, C. A. Gilbert, A. J. Lowrey, M. C. Callier, and C. E. Pandorf, "Long non-coding RNAs are transcriptional regulators of contractile protein-coding genes in skeletal muscle," The FASEB Journal, vol. 32, 2018.

[59] B. Li, H. Xu, H. Han et al., "Exosome-mediated transfer of lncRUNX2-AS1 from multiple myeloma cells to MSCs contributes to osteogenesis," Oncogene, vol. 37 , no. 41, pp. 5508 5519, 2018.

[60] E. Liapi, M. van Bilsen, R. Verjans, and B. Schroen, "tRNAs and tRNA fragments as modulators of cardiac and skeletal muscle function," Biochimica et Biophysica Acta (BBA)-Molecular Cell Research, vol. 1867, no. 3, p. 118465, 2020.

[61] J. Jehn and D. Rosenkranz, "tRNA-Derived Small RNAs: The Good, the Bad and the Ugly," Med One, vol. 4, no. 4, 2019.

[62] Y. Zhang, Y. Zhang, J. Shi et al., "Identification and characterization of an ancient class of small RNAs enriched in serum associating with active infection," Journal of Molecular Cell Biology, vol. 6, no. 2, pp. 172-174, 2014.

[63] S. R. Baglio, K. Rooijers, D. Koppers-Lalic et al., "Human bone marrow- and adipose-mesenchymal stem cells secrete exosomes enriched in distinctive miRNA and tRNA species," Stem Cell Research \& Therapy, vol. 6, no. 1, 2015.

[64] K. L. Inder, J. E. Ruelcke, L. Petelin et al., "Cavin-1/PTRF alters prostate cancer cell-derived extracellular vesicle content and internalization to attenuate extracellular vesicle-mediated 
osteoclastogenesis and osteoblast proliferation," Journal of Extracellular Vesicles, vol. 3, no. 1, 2014.

[65] C. P. Lai, O. Mardini, M. Ericsson et al., "Dynamic Biodistribution of Extracellular Vesicles in Vivo Using a Multimodal Imaging Reporter," ACS Nano, vol. 8, no. 1, pp. 483-494, 2013.

[66] M. Cataldi, C. Vigliotti, T. Mosca, M. R. Cammarota, and D. Capone, "Emerging Role of the Spleen in the Pharmacokinetics of Monoclonal Antibodies, Nanoparticles and Exosomes," International Journal of Molecular Sciences, vol. 18, no. 6, p. 1249, 2017.

[67] R. Narayanan, C.-C. Huang, and S. Ravindran, "Hijacking the cellular mail: exosome mediated differentiation of mesenchymal stem cells," Stem Cells International, vol. 2016, Article ID 3808674, 11 pages, 2016.

[68] W. Li, Y. Liu, P. Zhang et al., "Tissue-Engineered Bone Immobilized with Human Adipose Stem Cells-Derived Exosomes Promotes Bone Regeneration," ACS Applied Materials \& Interfaces, vol. 10, no. 6, pp. 5240-5254, 2018.

[69] M. Marrelli, C. Maletta, F. Inchingolo, M. Alfano, and M. Tatullo, "Three-point bending tests of zirconia core/veneer ceramics for dental restorations," International Journal of Dentistry, vol. 2013, Article ID 831976, 5 pages, 2013.

[70] M. Marrelli, A. Pujia, F. Palmieri et al., "Innovative approach for the in vitro research on biomedical scaffolds designed and customized with CAD-CAM technology," International Journal of Immunopathology and Pharmacology, vol. 29, no. 4, pp. 778-783, 2016.

[71] M. Marrelli, B. Codispoti, R. M. Shelton et al., "Dental pulp stem cell mechanoresponsiveness: effects of mechanical stimuli on dental pulp stem cell behavior," Frontiers in Physiology, vol. 9, 2018.

[72] S. Chen, Y. Tang, Y. Liu et al., "Exosomes derived from miR375-overexpressing human adipose mesenchymal stem cells promote bone regeneration," Cell Proliferation, vol. 52, no. 5, p. e12669, 2019.

[73] Z. F. Lu, Y. J. Chen, C. Dunstan, S. Roohani-Esfahani, and H. Zreiqat, "Priming Adipose Stem Cells with Tumor Necrosis Factor-Alpha Preconditioning Potentiates Their Exosome Efficacy for Bone Regeneration," Tissue Engineering Part A, vol. 23, no. 21-22, pp. 1212-1220, 2017.

[74] H. Li, D. Liu, C. Li et al., "Exosomes secreted from mutantHIF- $1 \alpha$-modified bone-marrow-derived mesenchymal stem cells attenuate early steroid-induced avascular necrosis of femoral head in rabbit," Cell Biology International, vol. 41, no. 12, pp. 1379-1390, 2017.

[75] F. Wei, M. Li, R. Crawford, Y. Zhou, and Y. Xiao, "Exosomeintegrated titanium oxide nanotubes for targeted bone regeneration," Acta Biomaterialia, vol. 86, pp. 480-492, 2019.

[76] J. M. Cooper, P. B. O. Wiklander, J. Z. Nordin et al., "Systemic exosomal siRNA delivery reduced alpha-synuclein aggregates in brains of transgenic mice," Movement Disorders, vol. 29, no. 12, pp. 1476-1485, 2014.

[77] T. Chen, F. Arslan, Y. Yin et al., "Enabling a robust scalable manufacturing process for therapeutic exosomes through oncogenic immortalization of human ESC-derived MSCs," Journal of Translational Medicine, vol. 9, no. 1, p. 47, 2011.

[78] L. Balaj, R. Lessard, L. Dai et al., "Tumour microvesicles contain retrotransposon elements and amplified oncogene sequences," Nature Communications, vol. 2, no. 1, 2011.

[79] M. D. Beckler, J. N. Higginbotham, J. L. Franklin et al., "Proteomic Analysis of Exosomes from Mutant KRAS Colon
Cancer Cells Identifies Intercellular Transfer of Mutant KRAS," Molecular Cellular Proteomics, vol. 12, no. 2, pp. 343-355, 2013.

[80] G. Spagnuolo, B. Codispoti, M. Marrelli, C. Rengo, S. Rengo, and M. Tatullo, "Commitment of Oral-Derived Stem Cells in Dental and Maxillofacial Applications," Dentistry Journal, vol. 6, no. 4, p. 72, 2018.

[81] A. Ballini, S. Cantore, S. Scacco, D. Coletti, and M. Tatullo, "Mesenchymal Stem Cells as Promoters, Enhancers, and Playmakers of the Translational Regenerative Medicine 2018," Stem Cells International, vol. 2018, Article ID 6927401, 2 pages, 2018.

[82] M. Tatullo, M. Marrelli, M. Amantea et al., "Bioimpedance Detection of Oral Lichen Planus Used as Preneoplastic Model," Journal of Cancer, vol. 6, no. 10, pp. 976-983, 2015.

[83] K. C. French, M. A. Antonyak, and R. A. Cerione, "Extracellular vesicle docking at the cellular port: Extracellular vesicle binding and uptake," Seminars in Cell \& Developmental Biology, vol. 67, pp. 48-55, 2017.

[84] A. Shimoda, Y. Tahara, S.-i. Sawada, Y. Sasaki, and K. Akiyoshi, "Glycan profiling analysis using evanescent-field fluorescence-assisted lectin array: Importance of sugar recognition for cellular uptake of exosomes from mesenchymal stem cells," Biochemical and Biophysical Research Communications, vol. 491, no. 3, pp. 701-707, 2017.

[85] L. Alvarez-Erviti, Y. Seow, H. F. Yin, C. Betts, S. Lakhal, and M. J. A. Wood, "Delivery of siRNA to the mouse brain by systemic injection of targeted exosomes," Nature Biotechnology, vol. 29, no. 4, pp. 341-345, 2011.

[86] J. Van Deun, P. Mestdagh, R. Sormunen et al., "The impact of disparate isolation methods for extracellular vesicles on downstream RNA profiling," Journal of Extracellular Vesicles, vol. 3, no. $1,2014$. 